\title{
Lentes progressivas - análise dos campos intermediário e de perto por deflexometria
}

\author{
Progressive addition lenses - analysis of intermediate and near vision zones by deflectometry
}

Celso Marcelo Cunhaํㄹ Renato José Bett Correia², Antonio Augusto Sardinha Neto ${ }^{3}$

\section{RESUMO}

Objetivo: Avaliar por deflexometria as diferentes regiões das lentes progressivas e determinar as áreas dos campos de visão intermediário e de perto.

Métodos: Foram incluídas vinte e duas lentes progressivas com poder +1,00 DE para longe e duas adições diferentes (adição 1,00 e 2,00, 11 de cada). Mediram-se as áreas dos campos intermediário e de perto entre as isoastigmáticas de 0,5 DC. Resultados: Encontraram-se diferenças significativas entre as áreas dos campos intermediário e de perto das lentes estudadas. Entre a área do campo intermediário e a adição observou-se correlação inversa; entre a área do campo intermediário e a extensão vertical do corredor encontrou-se correlação direta.

Conclusão: Com esses dados permitem-se recomendar as lentes de acordo com o campo de maior necessidade visual de cada usuário.

Descritores: Presbiopia/terapia; Lentes; Refração; Óptica e fotônica

\begin{abstract}
Purpose: To determine near and intermediate vision areas of progressive addition lenses by means of a deflectometer.

Methods: Twenty-two progressive addition lenses with +1.00 SD far power and two different additions (add 1.00 and 2.00; eleven subjects in each addition) were studied. Near and intermediatevision areas within 0.50 CD isoastigmatic lines were determined. Results: There are significant differences between near and intermediate vision areas of the studied lenses. There is also an inverse correlation between the addition and intermediate areas as well as direct relation between the vertical length of the corridor and its area.
\end{abstract}

Conclusion: Based on those findings, progressive addition lenses can be selected to suit the wearer's visual requirements.

Keywords: Presbyopia/therapy; Lenses; Refraction; Optics and photonics

\section{INTRODUÇÃO}

O Instituto Brasileiro de Geografia e Estatística no Censo 2010 estima que no Brasil existam cerca de 63 milhões (34\% da população) de pessoas acima de 40 anos $^{(1)}$. A presbiopia, sendo uma redução natural da capacidade acomodativa, está presente na maioria dos pacientes a partir da quinta década de vida ${ }^{(2)}$.

As lentes progressivas (LP), sem linha divisória entre os campos de visão de longe, intermediária e de perto, são largamente utilizadas para os présbitas amétropes ${ }^{(3)}$.

As LP têm passado por diversas etapas de evolução nos últimos 50 anos, porém, para ter os poderes dióptricos diferentes em cada campo, ainda mantêm áreas ao lado do corredor progressivo com aberrações em todas elas, chamadas de astigmatismo induzido (Al), ou também de astigmatismo irregular ou áreas de aberrações, em variáveis quantidades e distribuições ${ }^{(2,4)}$.

Existem vários fabricantes de LP, e mais de 150 modelos estão disponíveis no Brasil, no entanto, poucas informações técnicas sobre os Al são fornecidas aos oftalmologistas e ópticos. Existem poucos estudos sobre estas informações na literatura mundial, sendo a maioria estudos subjetivos baseados na satisfação dos pacientes ${ }^{(5)}$.

O objetivo deste estudo foi determinar as áreas da visão intermediária e de perto nas LP em duas adições distintas, por meio de um deflexômetro. A inadequação das áreas de perto e intermediária às necessidades visuais do portador poderia levar a não adaptação a lente escolhida.

\section{MÉTODOS}

Foram selecionadas 22 lentes progressivas das empresas Essilor ${ }^{\oplus}$, Multivis, Segment e Zeiss (Tabela 1). Todas com poder dióptrico de +1,00 esférico para longe; 11 com adição 1,00 (grupo 1) e 11 com adição 2,00 (grupo 2). Outras empresas do setor foram procuradas, mas não manifestaram interesse em incluir suas LP neste estudo (Lista das LP em anexo).

Foram solicitadas LP em resina surfaçadas para o olho direito, e selecionadas pela frequência que são encontradas em clínica privada e em hospital universitário de Cuiabá - MT, e por variedade de altura mínima de montagem. As LP foram nomeadas por letras de $A$ a $L$, sendo que as com adição 1,00 são acompanhadas do número 1, e as de adição 2,00 do número 2 .

As LP foram analisadas no deflexômetro de interferência de franjas "Rotlex Class Plus ${ }^{\circledR "}$ da Empresa Rotlex (1994) de Israel. Este instrumento detecta em segundos todas as medições dióptricas de uma lente em uma única medida. O aparelho utiliza uma fonte de "laser" puntiforme que incide diretamente sobre a lente examinada. Os raios refratados pela lente em avaliação passam por duas grades
Submetido para publicação: 24 de outubro de 2011 Aceito para publicação: 23 de novembro de 2012

Trabalho realizado no Hospital Geral Universitário, Cuiabá (MT), Brasil.

Médico, Oftalmocenter Santa Rosa, Cuiabá (MT), Brasil.

Médico, Hospital Geral Universitário, Cuiabá (MT), Brasil.

${ }^{3}$ Acadêmico de Medicina, Universidade Gama Filho, Rio de Janeiro (RJ), Brasil.
Financiamento: Não houve financiamento para este trabalho.

Divulgação de potenciais conflitos de interesse: C.M.Cunha, Nenhum; R.J.B.Correia, Nenhum; A.A.Sardinha Neto, Nenhum.

Endereço para correspondência: Renato José Bett Correia. Hospital Universitário Geral de Cuiabá. Rua 13 de junho, 2101 - Cuiabá (MT) - 78025-000

E-mail: celsomcunha@terra.com.br; renatobett@bol.com.br; sardinha_dm@hotmail.com Comitê de Ética: UNIC - 2011/048. 
Tabela 1. Dimensões de alguns parâmetros estudados nas lentes progressivas

\begin{tabular}{|c|c|c|c|c|}
\hline $\begin{array}{l}\text { Lentes } \\
\text { estudadas }\end{array}$ & $\begin{array}{l}\text { Comprimento do } \\
\text { corredor progressivo }\end{array}$ & $\begin{array}{c}\text { Distância da cruz de } \\
\text { montagem a 0,25 DE da adição }\end{array}$ & $\begin{array}{c}\text { Distância da cruz de montagem à linha } \\
\text { horizontal entre as marcas de remarcações }\end{array}$ & $\begin{array}{l}\text { Altura mínima de montagem } \\
\text { indicado pelo fabricante }\end{array}$ \\
\hline A1 & 8 & 6,0 & 3,8 & 18 \\
\hline B1 & 9 & 4,5 & 5,5 & 18 \\
\hline C1 & 7 & 2,5 & 2,7 & 16 \\
\hline D1 & 9 & 3,0 & 2,9 & 18 \\
\hline E1 & 8 & 2,5 & 3,7 & 20 \\
\hline F1 & 6 & 2,5 & 3,7 & 17 \\
\hline G1 & 7 & 3,5 & 3,7 & 18 \\
\hline $\mathrm{H} 1$ & 8 & 3,5 & 3,7 & 18 \\
\hline 11 & 8 & 4,0 & 4,0 & 17 \\
\hline J1 & 6 & 1,5 & 3,6 & 14 \\
\hline L1 & 8 & 4,0 & 4,0 & 17 \\
\hline A2 & 6 & 1,5 & 3,6 & 18 \\
\hline B2 & 6 & 1,5 & 5,5 & 18 \\
\hline$C 2$ & 5 & $-1,0$ & 2,7 & 16 \\
\hline D2 & 6 & 1,0 & 3,0 & 18 \\
\hline E2 & 6 & 1,0 & 4,0 & 20 \\
\hline F2 & 6 & 0,5 & 3,7 & 17 \\
\hline G2 & 6 & 0,5 & 3,7 & 18 \\
\hline $\mathrm{H} 2$ & 6 & 1,0 & 3,7 & 18 \\
\hline 12 & 6 & 1,8 & 3,8 & 17 \\
\hline$J 2$ & 5 & 0 & 3,6 & 14 \\
\hline L2 & 6 & 2,0 & 4,0 & 17 \\
\hline
\end{tabular}

Notas: Todas as medidas estão em milímetros.

Nas LP de adição 1,00 (A1 a L1) o corredor é da progressão de 0,25 a 0,75 DE. De adição 2,00 (A2 a L2) o corredor é da progressão de 0,75 a 1,50 DE.

Na coluna 3 o valor negativo representa o início de 0,25 DE da adição acima da cruz de montagem.

Altura mínima é a menor distância permitida pelo fabricante entre a cruz de montagem e a borda inferior da armação.

e formam um padrão tipo "moiré" em uma tela difusora. Destes se fazem mapas que representam o poder esférico, cilíndrico e eixo em cada milímetro da lente (Figura 1).

Os arquivos das 22 LP foram avaliados no "software" de análise do aparelho e registrados milimetricamente: na vertical da cruz de montagem a $22 \mathrm{~mm}$ abaixo; e, no sentido horizontal, $17 \mathrm{~mm}$ em cada lado da cruz de montagem.

Admitiram-se como limites superior e inferior do corredor progressivo:

- Nas lentes do grupo 1, poderes dióptricos esféricos entre +1,25 e $+1,75$ (valores compreendidos entre 0,25 e 0,75 da progressão desta adição).

- Nas lentes do grupo 2, poderes dióptricos esféricos entre +1,75 e $+2,50$ (valores compreendidos entre 0,75 e 1,50 da progressão desta adição).

Assim se procedeu pela necessidade de contar com 50\% a 75\% da adição para visão nítida na distância do campo de visão intermediária, pois alguma acomodação residual se observa nos usuários que necessitam das adições abaixo de 2,00.

Admitiu-se como limite superior da área de perto:

- Nas lentes do grupo 1, poderes dióptricos esféricos a partir de $+1,76$.

- Nas lentes do grupo 2, poderes dióptricos esféricos a partir de $+2,76$.

Admitiram-se como limites laterais do campo intermediário e de perto, em todas as lentes, as linhas isoastigmáticas de 0,50 DC (limite de Al).
As áreas de perto e intermediária foram calculadas em $\mathrm{mm}^{2}$ no "software" do deflexômetro.

Para avaliar a extensão horizontal mediu-se a largura do campo em três posições entre as isoastigmáticas de 0,50 DC no início, meio e fim do campo intermediário.

Para análise dos resultados adotou-se nível de significância de 5\% $(a=0,05)$, correspondente a probabilidade de erro $(p)<0,05$, valor considerado estatisticamente significativo. Empregou-se o teste estatístico não paramétrico Wilcoxon Signed Ranks Test para a correlação das adições. Para a correlação entre a altura do corredor progressivo e a área do campo intermediário utilizou-se a correlação linear de Pearson. Empregaram-se os programas Microsoft Excel 2000.

\section{RESULTADOS}

Os resultados gerais das medidas dos parâmetros das 22 LP estudadas se encontram na tabela 1.

A análise estatística com o Wilcoxon Signed Ranks Test mostra não haver concordância entre os parâmetros nas adições estudadas, logo as LP foram estudadas separadamente (grupo 1 para adição 1,00 e grupo 2 para adição 2,00)

No campo intermediário do grupo 1, encontra- se a mediana das áreas de 76,9 mm² e um alto coeficiente de variação (CV) de 30,2\%, conforme a tabela 2. Para o grupo 2, encontra-se a mediana das áreas de $20,7 \mathrm{~mm}^{2}$ e um médio para alto CV de 17,1\%, conforme a tabela 3.

A soma da extensão horizontal mostra o CV de 20 e 14,5\% para as LP dos grupos 1 e 2 , respectivamente. 
No campo de perto, quando se utiliza a altura mínima de montagem autorizada pelos fabricantes para cada uma das LP como limite inferior, encontra-se a mediana das áreas de 51,4 e 22,7 mm², e um

Anexo 1. Relação das lentes progressivas estudadas e seus fabricantes

\begin{tabular}{|c|c|c|}
\hline $\begin{array}{l}\text { Lentes } \\
\text { estudadas }\end{array}$ & $\begin{array}{c}\text { Nome das } \\
\text { lentes progressivas }\end{array}$ & $\begin{array}{c}\text { Fabricante das } \\
\text { lentes progressivas }\end{array}$ \\
\hline $\mathrm{A} 1$ & Espace Selective & Brasilor \\
\hline A2 & Espace Selective & Brasilor \\
\hline B1 & Gradal Top & Zeiss \\
\hline B2 & Gradal Top & Zeiss \\
\hline C1 & Multivis Ampla & Multivis \\
\hline C2 & Multivis Ampla & Multivis \\
\hline D1 & Multivis Extra & Multivis \\
\hline D2 & Multivis Extra & Multivis \\
\hline E1 & Sapphire LP & Segment \\
\hline E2 & Sapphire LP & Segment \\
\hline F1 & Sola Elan & Zeiss \\
\hline F2 & Sola Elan & Zeiss \\
\hline G1 & Solamax & Zeiss \\
\hline G2 & Solamax & Zeiss \\
\hline $\mathrm{H} 1$ & Varilux Comfort & Essilor \\
\hline $\mathrm{H} 2$ & Varilux Comfort & Essilor \\
\hline 11 & Varilux Physio & Essilor \\
\hline 12 & Varilux Physio & Essilor \\
\hline Ј1 & Varilux Pix & Essilor \\
\hline$J 2$ & Varilux Pix & Essilor \\
\hline L1 & Zeiss GT2 & Zeiss \\
\hline L2 & Zeiss GT2 & Zeiss \\
\hline
\end{tabular}

Obs:: As lentes progressivas 1 são adições 1,00 D e as lentes progressivas 2 são adiçōes 2,00 D. alto CV de 32,45 e 47,45\% para os grupos 1 e 2 respectivamente, conforme as tabelas 2 e 3 . Utilizando a altura de $22 \mathrm{~mm}$ como limite inferior fixo, encontra- se a mediana das áreas de 114,3 e 53,3 mm², e um alto CV de 21,5 e 26,7\% para os grupos 1 e 2 respectivamente, conforme as tabelas 2 e 3.

Existe correlação positiva forte entre a altura do corredor progressivo e a área do campo intermediário nas LP do grupo 1 (coeficiente de Pearson=0,92), e correlação positiva moderada nas LP do grupo 2 (coeficiente de Pearson=0,69).

\section{DISCUSSÃO}

Existem inúmeras combinações possíveis de curva base e desenho de LP. Alguns desenhos resultam em maior campo de longe, intermediário ou de perto. A presença concomitante dos três campos com grandes áreas e sem Al maior que 0,50 DC nas adições além de 1,50 parece não ser possível(6).

Neste estudo de LP com adições diferentes demonstrou-se relação diretamente proporcional entre o aumento do valor da adição e os Al, bem como relação inversa entre a altura do corredor e os Al, como relatado na literatura ${ }^{(7,8)}$. No entanto, no grupo 2, existe uma correlação linear moderada para esta segunda afirmativa, pois se considerou altura do corredor somente a porção de adição útil para a visão intermediária (entre 0,75 e 1,50 do total da adição). Encontrou-se a maioria dos corredores com $6 \mathrm{~mm}$ de altura. Acharam-se somente dois com $5 \mathrm{~mm}$, mas com um CV de 16,5\%, o que demonstra o empenho dos fabricantes no aumento da área intermediária, mas sem aumentar muito a altura do corredor.

Ressalta-se que os modelos mais atuais de LP são oferecidos com desenhos distintos pela variação das adições e mostram alturas verticais totais dos corredores (desde 0,25 até 1,75 da adição 2,00) maiores com o aumento da adição, deixando as LP de adição 2,00 com Al mais reduzido do que se fosse mantida a altura dos corredores das adições 1,00.

O limite do Al tolerável pelos pacientes tem sido estudado na literatura. Alguns autores, em estudos clínicos, encontraram 0,50 DC e outros até 1,00 DC sem alterar a AV ou aumentar significativamente as aberrações de maior ordem. Como a maioria dos estudos objetivos utilizou 0,50 DC, manteve-se este parâmetro para poder comparar as
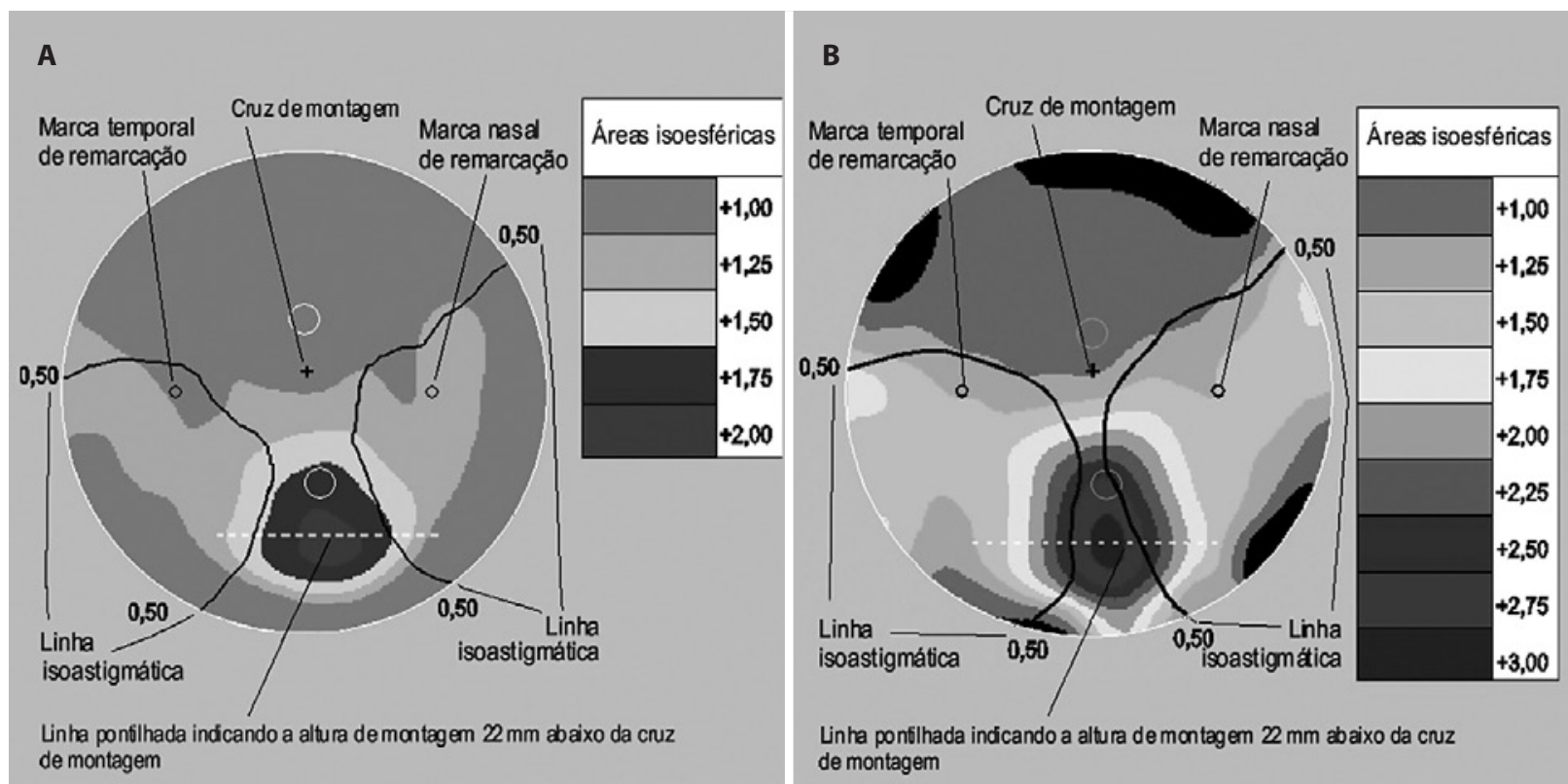

Figura 1. Mapas de contornos por deflexometria vistos de frente. Áreas isoesféricas e linhas isoastigmáticas de 0,50 DC. A) LP D1; B) LP D2. 
Tabela 2. Áreas dos campos intermediário e de perto e altura do campo de perto nas LP do grupo 1

\begin{tabular}{|c|c|c|c|c|c|c|}
\hline $\begin{array}{l}\text { Lentes } \\
\text { estudadas }\end{array}$ & $\begin{array}{c}\text { Área do campo } \\
\text { intermediário com } \\
\text { progressão da adição } \\
\text { de } 0,25 \text { a } 0,75 \mathrm{DE} \\
\text { com isoastigmática } \\
\text { limite de } 0,50 \mathrm{DC}\end{array}$ & $\begin{array}{c}\text { Soma das } \\
\text { extensões laterais } \\
\text { das três barras } \\
\text { horizontais } \\
\text { do campo } \\
\text { intermediário }\end{array}$ & $\begin{array}{c}\text { Área do campo } \\
\text { de perto com progressão } \\
\text { de } 0,76 \text { da adição e } \\
\text { com limite inferior } \\
\text { de } 22 \mathrm{~mm} \text { de altura } \\
\text { de montagem }\end{array}$ & $\begin{array}{c}\text { Área do campo perto } \\
\text { com progressão de } 0,76 \\
\text { da adição e com limite } \\
\text { inferior na altura mínima } \\
\text { de montagem de cada } \\
\text { LP (fabricante) }\end{array}$ & $\begin{array}{l}\text { Altura da cruz de } \\
\text { montagem ao } \\
\text { início do campo } \\
\text { de perto } \\
(0,76 \mathrm{DE} \text { da } \\
\text { adição) }\end{array}$ & $\begin{array}{l}\text { Altura da área } \\
\text { do campo de } \\
\text { perto com a } \\
\text { altura mínima } \\
\text { de montagem } \\
\text { de cada LP }\end{array}$ \\
\hline A1 & 76,9 & 29,9 & 82,6 & 31,9 & 15,0 & 4 \\
\hline B1 & 94,1 & 36,0 & 72,4 & 39,1 & 14,5 & 4 \\
\hline $\mathrm{C} 1$ & 63,0 & 27,9 & 104,1 & 45,8 & 10,7 & 6 \\
\hline D1 & 119,6 & 42,6 & 116,1 & 54,9 & 13,0 & 6 \\
\hline E1 & 65,1 & 25,7 & 94,8 & 80,0 & 10,7 & 10 \\
\hline F1 & 43,4 & 22,2 & 124,6 & 70,3 & 9,7 & 8 \\
\hline G1 & 56,7 & 25,1 & 141,6 & 89,3 & 11,7 & 7 \\
\hline $\mathrm{H} 1$ & 80,3 & 32,2 & 124,1 & 73,8 & 12,7 & 6 \\
\hline 11 & 81,7 & 32,9 & 114,3 & 46,4 & 13,0 & 5 \\
\hline J1 & 45,2 & 23,6 & 152,5 & 51,4 & 8,6 & 6 \\
\hline L1 & 77,1 & 30,0 & 101,6 & 44,1 & 13,0 & 5 \\
\hline Mediana & 76,9 & 29,9 & 114,3 & 51,4 & 12,7 & 6 \\
\hline $\begin{array}{l}\text { Coeficiente de } \\
\text { variação (CV) }\end{array}$ & $30,2 \%$ & $20,0 \%$ & $21,5 \%$ & $32,5 \%$ & $16,4 \%$ & $28,9 \%$ \\
\hline
\end{tabular}

Notas: Áreas medidas em mm². Extensão horizontal e altura em mm.

Tabela 3. Áreas dos campos intermediário e de perto e altura do campo de perto nas LP do grupo 2

\begin{tabular}{|c|c|c|c|c|c|c|}
\hline $\begin{array}{l}\text { Lentes } \\
\text { estudadas }\end{array}$ & $\begin{array}{c}\text { Área do campo } \\
\text { intermediário com } \\
\text { progressão da adição } \\
\text { de } 0,75 \text { a } 1,50 \mathrm{DE} \text { com } \\
\text { isoastigmática limite } \\
\text { de } 0,50 \mathrm{DC}\end{array}$ & $\begin{array}{c}\text { Soma das } \\
\text { extensões laterais } \\
\text { das três barras } \\
\text { horizontais } \\
\text { do campo } \\
\text { intermediário }\end{array}$ & $\begin{array}{l}\text { Área do campo } \\
\text { de perto com } \\
\text { progressão de } 1,75 \text { da } \\
\text { adição e com limite } \\
\text { inferior de } 22 \text { mm de } \\
\text { altura de montagem }\end{array}$ & $\begin{array}{l}\text { Área do campo perto } \\
\text { com progressão de } \\
\text { 1,75 da adição e com } \\
\text { limite inferior na altura } \\
\text { mínima de montagem } \\
\text { de cada LP (fabricante) }\end{array}$ & $\begin{array}{l}\text { Altura da cruz de } \\
\text { montagem ao } \\
\text { início do campo } \\
\text { de perto } \\
(1,75 \mathrm{DE} \text { da } \\
\text { adição) }\end{array}$ & $\begin{array}{l}\text { Altura da área } \\
\text { do campo de } \\
\text { perto com a } \\
\text { altura mínima de } \\
\text { montagem } \\
\text { de cada LP }\end{array}$ \\
\hline $\mathrm{A} 2$ & 24,3 & 17,8 & 53,0 & 22,7 & 15,6 & 3 \\
\hline B2 & 22,8 & 15,7 & 47,0 & 25,7 & 15,5 & 4 \\
\hline$C 2$ & 18,2 & 14,8 & 53,3 & 21,8 & 10,7 & 6 \\
\hline D2 & 23,6 & 18,5 & 41,3 & 12,2 & 16,0 & 3 \\
\hline E2 & 18,5 & 13,7 & 54,7 & 41,0 & 15,0 & 6 \\
\hline F2 & 19,5 & 14,5 & 63,9 & 28,9 & 13,7 & 4 \\
\hline G2 & 18,4 & 15,4 & 93,3 & 45,8 & 13,0 & 6 \\
\hline $\mathrm{H} 2$ & 20,7 & 14,7 & 75,0 & 37,2 & 13,7 & 5 \\
\hline 12 & 24,6 & 16,9 & 52,6 & 17,0 & 14,8 & 3 \\
\hline 12 & 13,2 & 10,4 & 70,8 & 16,7 & 11,6 & 3 \\
\hline L2 & 24,2 & 14,1 & 42,2 & 8,6 & 16,0 & 2 \\
\hline Mediana & 20,7 & 14,8 & 53,3 & 22,7 & 14,8 & 4 \\
\hline $\begin{array}{l}\text { Coeficiente de } \\
\text { variação }(C V)\end{array}$ & $17,1 \%$ & $14,5 \%$ & $26,7 \%$ & $47,4 \%$ & $12,7 \%$ & $35,3 \%$ \\
\hline
\end{tabular}

Notas: Áreas medidas em mm². Extensão horizontal e altura em mm.

$L P^{(4,7,9)}$. Este limite de 0,50 DC é uma das linhas isoastigmáticas que compõe o mapa de contornos cilíndricos das LP (Figura 1).

A evolução das LP, nos últimos anos, tem se concentrado no aumento lateral da área do corredor com Al mínimo para facilitar o uso do computador, uma das atividades que mais necessitam da visão no campo intermediário. O monitor é focalizado com 50\% e 75\% do valor total da adição $(0,50$ e 0,75 DE para adição 1,00; e entre 1,00 e
1,50 DE para adição 2,00)(10,11). Admite-se que o tamanho da área intermediária por si só não garanta o melhor desempenho destas lentes, pois, por exemplo, pode-se ter um mesmo tamanho de área em duas LP: uma com altura de $10 \mathrm{~mm}$ e $4 \mathrm{~mm}$ de largura; e outra de $4 \mathrm{~mm}$ de altura e $10 \mathrm{~mm}$ de largura. No entanto, é bem provável que a LP com maior largura de corredor facilite o desempenho da leitura, pois, para esta tarefa, deve-se ter mais campo horizontal do que vertical. 
Para comparar a extensão horizontal das áreas dos campos intermediários utilizou-se a soma da largura de três posições horizontais neste estudo (Tabelas 2 e 3), mostrando um CV moderado de 20,2 e 14,5\% para o grupo 1 e 2 respectivamente, o que demonstra a dificuldade dos fabricantes do setor em manter um campo maior, de pouco Al, nas adições acima de 2,00.

Estudo semelhante na literatura americana ${ }^{(7)}$ utilizou 28 LP, três delas incluídas neste estudo (as LP B 2, G 2 e H 2). Observa-se que estas LP mantêm as proporções dos tamanhos entre as áreas dos campos intermediário e de perto. No entanto, o estudo citado considerou somente uma largura horizontal para comparar a extensão lateral do campo intermediário e, para área considerou duas vezes o componente horizontal por uma vez do vertical.

Por sua vez, a tendência dos fabricantes ao aumento do campo intermediário (lentes progressivas A2, C2, D2, I2 e L2), vem reduzindo o campo de perto, quando estas lentes são montadas com a altura mínima sugerida pelos fabricantes, principalmente nas lentes com adições maiores (Tabela 3).

Na literatura, encontra-se comparação da área do campo de perto entre algumas lentes em alturas fixas $(16,18,22 \mathrm{~mm})$ para todas elas, de forma justa a compará-las ${ }^{(4,7)}$. No entanto, isto não ocorre na prática, em razão da variação da altura mínima sugerida para cada tipo de LP pelos fabricantes. Neste estudo, verificaram-se também as alturas variáveis para compará-las na forma que se encontram disponíveis aos usuários.

Dentre as lentes do grupo 1, as cinco com maiores áreas em ordem decrescente são:

Área intermediária: D1, B1, I1, H1 e L1.

Área de perto (aplicando-se como limite inferior a altura mínima de montagem sugerida pelo fabricante): G1, E1, H1, F1 e D1.

Dentre as lentes do grupo 2, as cinco com maiores áreas em ordem decrescente são:

Área intermediária: 12, A2, L2, D2 e B2.

Área de perto (aplicando como limite inferior a altura mínima de montagem sugerida pelo fabricante): G2, E2, H2, F2 e B2.

Os achados sugerem a conveniência de se basear a recomendação das LP com base nos tamanhos das áreas de visão intermediária e de perto as relacionando à necessidade visual do paciente. Alguns estudos têm se ocupado deste aspecto ${ }^{(4,7)}$, no entanto, necessitam-se ensaios clínicos para sua melhor compreensão. O presente estudo tratou apenas de um dos aspectos relacionados às LP. Outros fatores importantes, tais como simetria dos desenhos, poder total do $\mathrm{Al}$, aberrações de segunda ordem, equilíbrio binocular e indução prismática podem influir sobre a adaptação e desempenho visual do portador.

Nas tabelas 2 e 3, constata-se a independência entre o tamanho da área de perto e o Al, dessa maneira, corroborando os achados da li- teratura ${ }^{(7)}$. Por outro lado, observa-se a correlação positiva forte entre altura do corredor e área do campo intermediário no grupo 1 ( $r$ : 0,92) e correlação positiva moderada no grupo 2 (r: 0,69), demonstrando os esforços dos fabricantes para desenvolverem as LP, como já citado anteriormente. Por este fato, o surgimento de LP com corredor mais curto para armações pequenas, com altura mínima de montagem de 14 mm, não é considerado, pelos autores, como evolução das LP, e sim adaptação dos fabricantes a tais armações. Isto, talvez, até não seja um problema nas LP de adição 1,00, porém, naquelas do grupo 2 (LP J2=área intermediária de 13,2 $\mathrm{mm}^{2}$ - mediana deste grupo=20,7), deve levar a menor rendimento visual neste campo.

\section{CONCLUSÃO}

Encontraram-se diferenças estatisticamente significativas entre as áreas dos campos intermediário e de perto das diferentes lentes progressivas. Seu conhecimento oferece subsídios aos oftalmologistas para orientarem os pacientes na escolha de lentes adequadas a suas necessidades visuais. Porém, novos estudos são necessários para comprovar esta hipótese clínica.

\section{AGRADECIMENTOS}

Agradecemos ao Sr. Gilmar Oliveira Júnior pelo apoio estatístico.

\section{REFERÊNCIAS}

1. Instituto Brasileiro de Geografia e Estatística - IBGE. Censo Demográfico 2010. Disponível: <http://www.censo2010.ibge.gov.br/sinopse/webservice/>. Acesso em 04 de maio de 2011.

2. Lui Netto A, Alves MR, Lui AC, Lui GA, Giovedi Filho R, Lui TA, et al. Avaliação clínica comparativa de lentes progressivas na correção da presbiopia. Rev Bras Oftalmol. 2009;68(3):129-33.

3. Karp A. Lenses by the numbers. Lenses Technol. 2004;50-4

4. Sheedy J, Hardy RF, Hayes JR. Progressive addition lenses--measurements and ratings. Optometry. 2006;77(1):23-39.

5. Monte FQ, Carvalho Filho CJ. Proposta para uma visão clínica das lentes progressivas. Rev Bras Oftalmol. 2008;67(2):69-81.

6. Minkwitz G. [On the surface astigmatism of a fixed symmetrical aspheric surface]. Opt Acta (Lond). 1963;10:223-7. German.

7. Sheedy JE. Correlation analysis of the optics of progressive addition lenses. Optom Vis Sci. 2004;81(5):350-61.

8. Bell GR. Verifying and evaluating progressive addition lenses in clinical practice. Optometry. 2001;72(4):239-46.

9. Selenow A, Bauer EA, Ali SR, Spencer LW, Ciuffreda KJ. Assessing visual performance with progressive addition lenses. Optom Vis Sci. 2002;79(8):502-5.

10. Sheedy JE, Shaw-McMinn PG. Diagnosing and treating computer-related vision problems. Woburn, Mass: Butterworth-Heinemann; 2002.

11. Pedrono C, Obrecht G, Stark L. Eye-head coordination with laterally "modulated" gaze field. Am J Optom Physiol Opt. 1987;64(11):853-60. 